\title{
FIRE SPREAD PREDICTION USING PROBABILISTIC CELLULAR AUTOMATA: THE CASE OF URBAN SETTLEMENTS IN THE PHILIPPINES
}

\author{
E. E. Tan ${ }^{1, *}$, A. J. Vicente ${ }^{1, *}$ \\ ${ }^{1}$ FireCheck Project - University of the Philippines Cebu, Cebu, Philippines - (eltan2, aovicente)@up.edu.ph
}

\section{Commission IV}

KEY WORDS: fire spread modeling, probabilistic cellular automata, disaster and risk reduction management, geographic information systems

\begin{abstract}
:
Fire disasters are common occurrences in the urban settlements of the Philippines. Concerned agencies like the Bureau of Fire Protection (BFP) and the Disaster and Risk Reduction Management Office (DRRMO) are constantly planning ways to prevent and mitigate fire disasters. The key to an effective plan against fire disaster is understanding how a potential fire can spread in a community. By combining both GIS and Probabilistic Cellular Automata (PCA), this paper solves the task of fire spread modeling and simulation. PCA is a model that consists of a regular grid of cells, whose cells are updated according to rules that take into account both the cell's current state and the cell's neighbors' states. The model we developed factors in wind, building materials, and building density. The model was designed after several fires in major cities of Cebu, Philippines. An accuracy of $83.54 \%$ and a Cohen's Kappa coefficient of 0.67 was achieved. Further, a web-based tool was developed to aid in fire disaster planning.
\end{abstract}

\section{INTRODUCTION}

Urban fires are a far too common occurrence in the Philippines. From January to November of 2018, the Bureau of Fire Protection - Region 7 (BFP 7) has recorded 550 fire incidents-all in Cebu province alone (Galarpe, 2019). Although these fire incidents are very common, steps haven't been taken to aid the response of the local government units (LGUs) when a fire breaks out. Furthermore, evacuation plans are either missing or non-standardized. Hence, to aid in disaster and the LGU's disaster and risk reduction management (DRRM), we would like to understand how a fire spreads in an urban setting. The model presented in this paper is a step towards understanding urban fire, and we hope it would help inform the decision making of each LGU's DRRM teams.

Fire is a very complicated phenomenon; hence, its spread is quite difficult to accurately capture and model. This is due to its inherent erratic behavior, which introduces a level of uncertainty when capturing and modeling this phenomenon. Hence the model used in this paper also utilizes some form of randomness, which makes repeat simulations yield slightly different results.

There already have been studies regarding urban fire spread modeling using Cellular Automata. However, most of these studies are set in Japan, of which might not directly translate to the Philippine urban setting due to differences in building materials, setup, and density. This is due to the many shanties that are clumped densely together in the city, with access roads far too narrow for fire trucks to reach and building materials far lighter than should be. Therefore this paper aims to adapt previous work in fire spread modeling and apply it to the case of the Philippines' urban settlements.

\footnotetext{
* Corresponding author
}

\section{CELLULAR AUTOMATA}

Cellular automata is a type of computer model that consists of a grid of cells, wherein each cell can be one of a finite number of states. These cell states can be updated using rules that determine the new state of the cell. Each cell is also aware of their neighboring cells which we call, quite simply, the neighborhood.

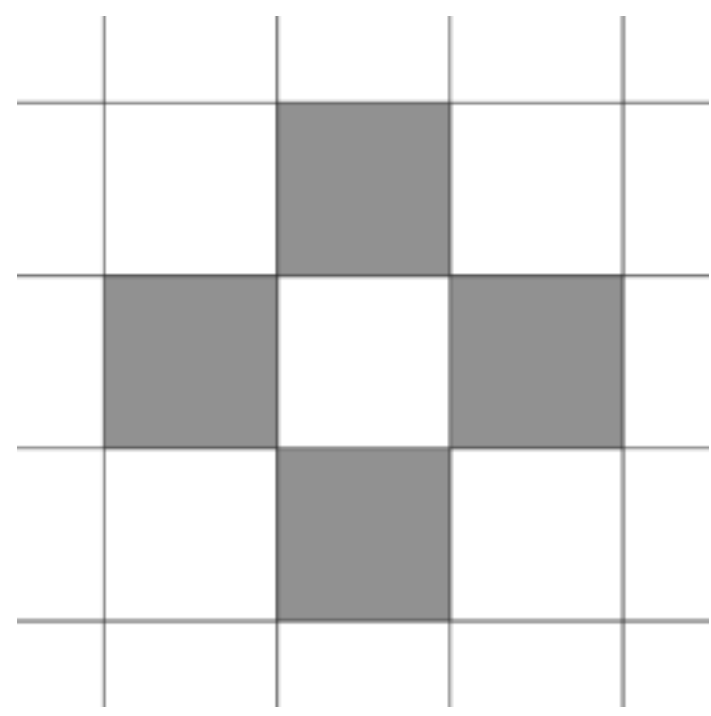

Figure 1: von Neumann neighborhood of $r=1$

A cell's neighborhood can be defined as one of many types, with the von Neumann neighborhood being the most popular. The von Neumann neighborhood is composed of a central cell's adjacent cells within a Manhattan distance of $r=1$ (Figure 1). This type of neighborhood is easily extended by 
increasing the radius by which we consider the neighborhood (Figure 2). The model used in this paper utilizes this type of neighborhood.

Cellular automata models are a good fit for the modeling of physical systems composed of interacting components (Chopard, 2012). These models have been used to model various fields of Physics such as diffusion systems, of which fire is an instance. Moreover, cellular automata have also been used to model pattern formation, fluid flow, and road traffic.

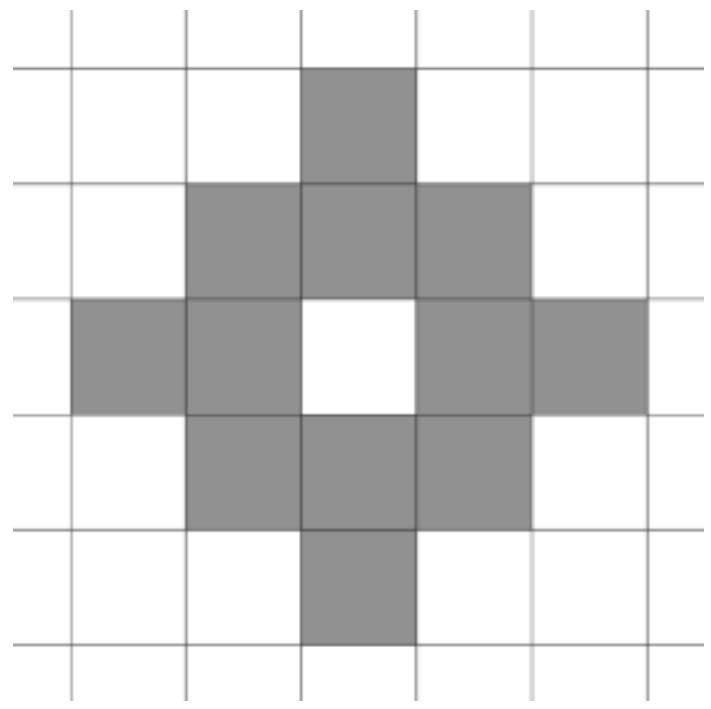

Figure 2: von Neumann neighborhood of $r=2$

Models utilizing cellular automata can also be run in parallel, potentially reducing the time required for simulating a scenario.

\subsection{Probabilistic Cellular Automata}

Probabilistic Cellular Automata (PCA), also called Stochastic Cellular Automata, are a type of cellular automata that utilizes an update rule that relies on randomness. This type of cellular automata is appropriate for modeling fire due to the complexity involved in fire spread. Since we are unable to accurately and completely capture the behavior of fire, we have to resort to stochasticity to capture the unpredictable behavior of fire. The probabilistic update rule is hence an attempt to factor in and capture the unpredictability of fire.

PCA have been used to model several phenomena similar to fire spread; further establishing the feasibility of using PCA for urban fire spread. Studies like Brieger and Bonomi's (1991) successfully used PCA to model a non-linear diffusion equation, successfully simulating diffusion on a macroscale. Bhargava et al. (1993) were able to use PCA in modeling innovation adoption in a population, where innovators influence their neighbors to adopt a new product. Almeida and Macau (2011) used PCA to model wildland fires, where they found that it sufficiently constitutes a qualitative framework for wildland fire spread simulation. Additionally, Ohgai et al. (2007) developed a PCA model for a built-up urban area of Japan. This study by Ohgai is the model adopted for use in this paper.

\section{FIRE SPREAD MODELING}

Most of the previous studies on fire spread modeling have focused on forest fires (Carmel et al., 2009; Karafyllidis and
Thanailakis, 1997; Sullivan, 2009). Meanwhile, studies on urban fire spread are focused more on simulating fire spread based on physics or on the microscale (Himoto and Tanaka, 2008; Lee and Davidson, 2010); however, physics based models are computationally heavy and require a considerable amount of time to simulate. In contrast to physics based models, cellular automata models are computationally easier due to the amount of approximation performed. The following sections will discuss the modeling process.

\subsection{Cell Size}

The cell size of the grid defines how detailed the simulated fire would be. A large cell size will yield a less precise simulation but will be computationally faster. On the other hand, a smaller cell size will increase simulation detail but require more computation to cover the same area.

$$
D=1.15(5+0.5 v)
$$

Equation 1 shows an equation proposed by Hamada (as cited by Ohgai et al. (2007)) that represents the maximum distance that a fire can spread. With a wind velocity $v$ of 0 , this suggests the limit that fire can spread is $5.75 \mathrm{~m}$. Hence, a cell size greater than $5.75 \mathrm{~m}$ would be insufficient in capturing the spread of fire. A cell size of $3 \mathrm{~m} \times 3 \mathrm{~m}$ was chosen by Ohgai et al. (2007) since it's approximately half of the limit $(5.75 \mathrm{~m})$, which should capture situations of wind and no wind appropriately. The same cell size of $3 \mathrm{~m} \times 3 \mathrm{~m}$ is adopted in this paper.

\subsection{Attributes}

The following attributes are utilized in the model:

$\begin{array}{ll}\text { 1. } & \text { Building material } \\ \text { 2. } & \text { Building height } \\ \text { 3. } & \text { Wind speed } \\ \text { 4. } & \text { Wind direction }\end{array}$

The building attributes influence the probability of burning, while the wind attributes influence the speed and direction of the fire spread. Building attributes were collected by enumerators hired by the FireCheck project, while wind attributes are sourced from timeanddate.com.

\subsection{Polygon to Cell Conversion}

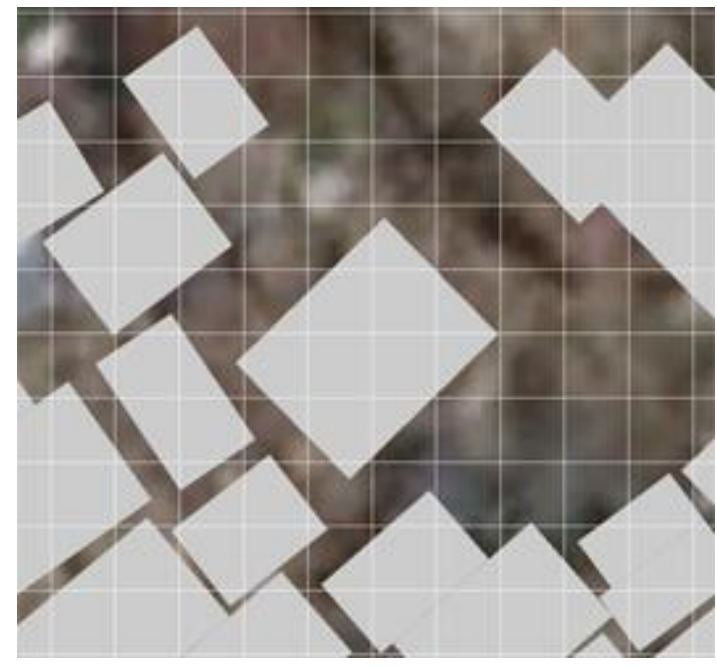

Figure 3: Grid overlaid on top of polygons 
A grid of cells is created and overlaid on top of digitized polygons as shown in Figure 3. Using the data collected by the FireCheck Project, the digitized polygons are attributed with the structure material and height. The data contained in these attributed polygons are transferred to cells in a grid by way of a spatial join with a buffer of $-0.8 \mathrm{~m}$ (as shown in Figure 4). That is, a polygon that overlaps with a negative $0.8 \mathrm{~m}$ buffered cell will have their attributes copied over to the cell. Additionally, a cell that does not overlap with any polygon is assigned as an empty cell; this type of cell has a burn probability of $0 \%$. This allows us to represent dense structures more sufficiently than without a buffer; otherwise, small spaces in between dense structures will be misrepresented as buildings.

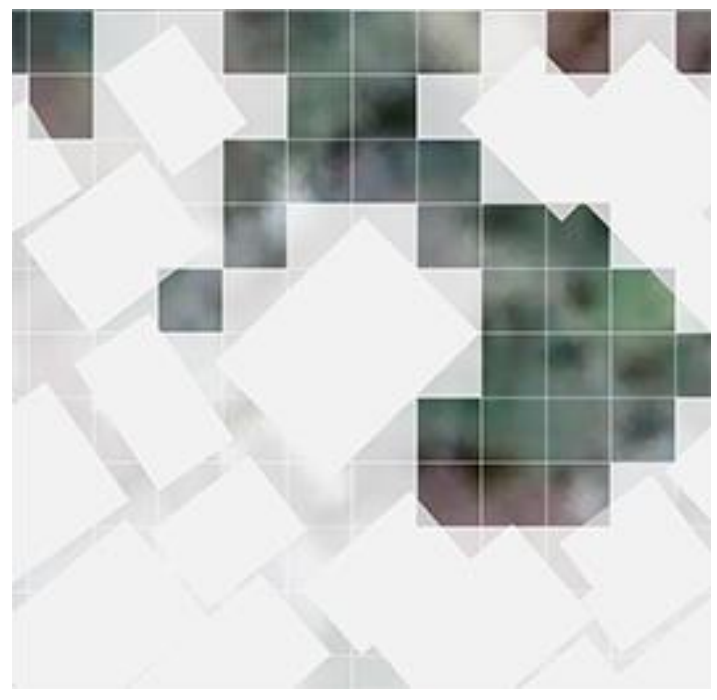

Figure 4: Cells selected with a buffer of $-0.8 \mathrm{~m}$ in white

\subsection{Neighbors}

The size of the neighborhood of a cell is determined by the same equation previously proposed by Hamada (eq. 1). Hence, wind speed determines the size of the neighborhood per simulation.

\begin{tabular}{ll}
$\mathrm{v}$ & $\mathrm{D}$ \\
\hline 0 & 5.75 \\
1 & 6.325 \\
2 & 6.9 \\
3 & 7.475 \\
4 & 8.05 \\
5 & 8.625 \\
6 & 9.2
\end{tabular}

Table 1: Limit of fire spread $(\mathrm{m})$ for wind speed $(\mathrm{m} / \mathrm{s}) v=0$ through 6

As can be seen in Table 1, when wind speed is 0 , the limit that fire can spread is at $5.75 \mathrm{~m}$. Since a von Neumann neighborhood size of 2 would cover at least $6 \mathrm{~m}$ and at most $7.5 \mathrm{~m}$, a cell size of $3 \mathrm{~m} \times 3 \mathrm{~m}$ is appropriate for cases of no wind. Hence, the smallest neighborhood size that we should consider is of size 2 .

\subsection{Cell States}

\begin{tabular}{ll} 
State & Description \\
\hline 0 & Unburnable/No structure \\
1 & Burnable \\
2 & Ignited, no ability to spread fire \\
3 & Burning, possess ability to spread fire \\
4 & Burned out, no ability to spread fire \\
& Table 2: Possible cell states
\end{tabular}

There are 5 possible cell states that represent the spread of fire in this model. Cell states can only transition to higher states, from state [1] to state [4]. State [0] is unburnable and does not represent any structure; this state does not change. A cell in state [1] has the potential to burn and will transition to state [2] once catching fire. Cells in state [2] have caught fire, but these cells are not able to spread the fire to its neighboring cells. After a predetermined amount of time $t_{1}$, cells in state [2] transition to state [3]. Cells in state [3] are on fire, and these cells are able to spread fire to their neighbors. Finally, these cells in state [3] burn for a time $t_{2}$ until after which they burn out and transition to state [4]. Times $t_{1}$ and $t_{2}$ will be elaborated in the next section. State [4] is the burned out state, cells with this state are no longer able to spread fire. Table 2 summarizes these 5 states.

\subsection{Fire Spreading Probability}

The deciding factor of when a cell with state [1] transitions to state [2] is dependent on a probability $F$.

$$
F=\alpha \cdot S \cdot p(T) \cdot W
$$

Where $\alpha$ is a constant, $S$ is the building structure parameter, $p$ is heat parameter, $T$ is the duration parameter, and $W$ is the wind parameter.

$\alpha$ is a global constant that influences the speed at which the fire spreads, this potentially allows us to decrease the overall speed of the fire due to other factors such as rain. $S$ is based on the type of building material, which determines the propensity of the cell to ignite on fire. Using the data collected by the FireCheck Project, there are 4 types of materials (cement, metal, wood, and plastic). Cells with the cement material have their $S$ attribute set to 0.01 , metal cells are set to 0.2 , and both wood and plastic are set to 1 . In the case of mixed material (as in the case of cells having both cement and wood), cells have their $S$ set to 0.7 .

$$
p(T)=\left\{\begin{array}{l}
\frac{4.0}{t_{2}-t_{1}} \cdot T+\frac{0.2 \cdot t_{2}-4.2 \cdot t_{1}}{t_{2}-t_{1}} \quad\left[t_{1} \leq T \leq \frac{t_{2}-t_{1}}{5}+t_{1}\right] \\
\frac{5}{4 \cdot\left(t_{2}-t_{1}\right)} \cdot\left(-T+t_{2}\right) \quad\left[\frac{t_{2}-t_{1}}{5}+t_{1} \leq T \leq t_{2}\right]
\end{array}\right.
$$

$p$, as defined in Equation 3 is a formula developed by Wakamatsu (as cited by Ohgai et al. (2007)); this formula is based on the indoor temperature of a wooden house on fire. $p$ is dependent on $T$, which allows us to model the changes in heat released as time passes. As introduced in the previous section, $t_{1}$ and $t_{2}$ are the spread delay time and max burn time, respectively. The spread delay time is the time between a cell being ignited to being able to spread fire to its neighbors. Based on a model experiment on wooden $3.6 \mathrm{~m} \times 3.6 \mathrm{~m}$ houses by Himoto et al. (2018), they found that the median time for a house to start spilling smoke to eventually ejecting flame is 220 s. Hence, we will set $t_{1}$ to the closest minute of 4 . The max 
burn time is the time from when a cell is ignited to being completely burned down, no longer able to spread fire. Using the same data from Himoto et al. (2018), we find that the median time between the ignition of the house to the ultimate collapse of the roof is $595 \mathrm{~s}$. Therefore, we also set $t_{2}$ to the closest whole minute, which is 10 . Finally, $W$ influences the direction of the fire spread by providing a bias to cells in the downwind direction. This is done by creating $90^{\circ}$ cones that originate from cells that are considered on fire, with their opening towards the downwind direction.

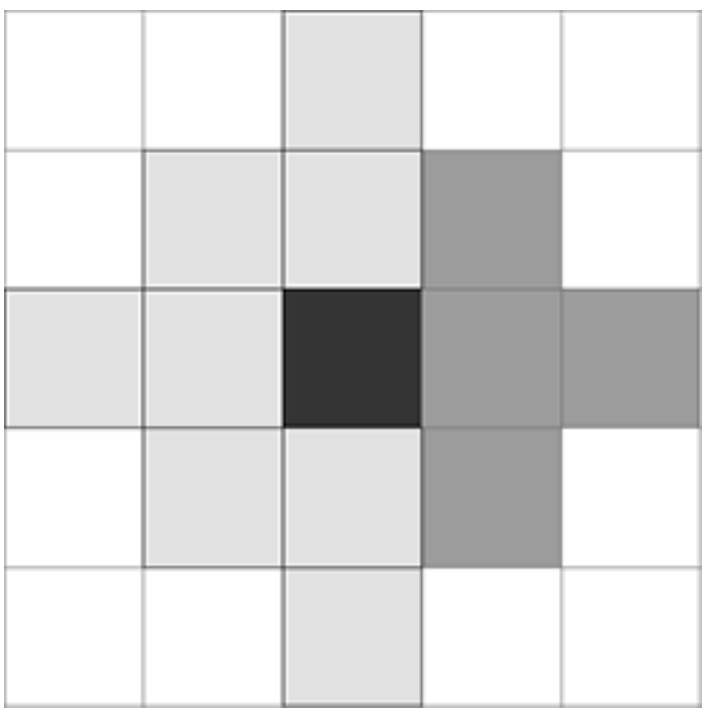

Figure 5: Neighbors given positive bias (middle gray) in easterly wind, fire origin (black), and neighbors given negative bias (light gray)

Figure 5 shows the case of an easterly wind where the east side of the fire origin is selected to have a higher bias with the formula:

$$
W=\sqrt{\frac{3}{d_{o}}}
$$

where $d_{o}$ is the distance of the cell from the origin (m). Cells upwind are given a negative bias with the following formula:

$$
W=\frac{1.5}{d_{o}}
$$

\subsection{Simulation Process}

Provided with the simulation extent, fire origin, wind parameters, and building parameters, the simulation process is described below.

- Step 1: A grid is created and overlaid on top of the simulation extent; the material and building type is copied over from the attributed polygons to their mapped cells.

- Step 2: Multiple matrices are created to represent the different parameters used for probability calculation (Equation 2).

The following matrices are created in way that each element $(i, j)$ refers to a single cell.

\section{- Duration}

- Flammability

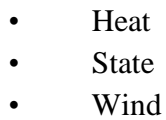

The duration matrix keeps track of how long a cell $(i, j)$ has been on fire in minutes. The flammability matrix is set based on the material type as previously discussed. The heat matrix is calculated using Wakamatsu's equation (3) from the current grid; only cells that have their burn duration $>t_{1}$ are included in the calculation. The state matrix holds the current state of each cell. The wind matrix stores the influence of wind for each cell.

- Step 3: Wind and heat parameter is calculated from the current grid.

- $\quad$ Step 4: Probability $F$ (Equation 2) is calculated and tested; cells in state [1] that satisfy the probability are transistioned to state [2] (Table 2).

- $\quad$ Step 5: Based on $t_{1}$ and $t_{2}$, cells are advanced to their respective states; cells in state [2] with duration $>t_{1}$ are advanced to state [3], while cells in state [3] with duration $>t_{2}$ are retired to state [4].

- Step 6: Duration for cells with duration $>1$ is incremented by 1 ; every iteration of the simulation is set to 1 minute.

- $\quad$ Step 7: Repeat steps 3 - 6 until a predefined end time.

\subsection{Simulation Platform}

The utilization of this model is simplified for use through the creation of a user interface.

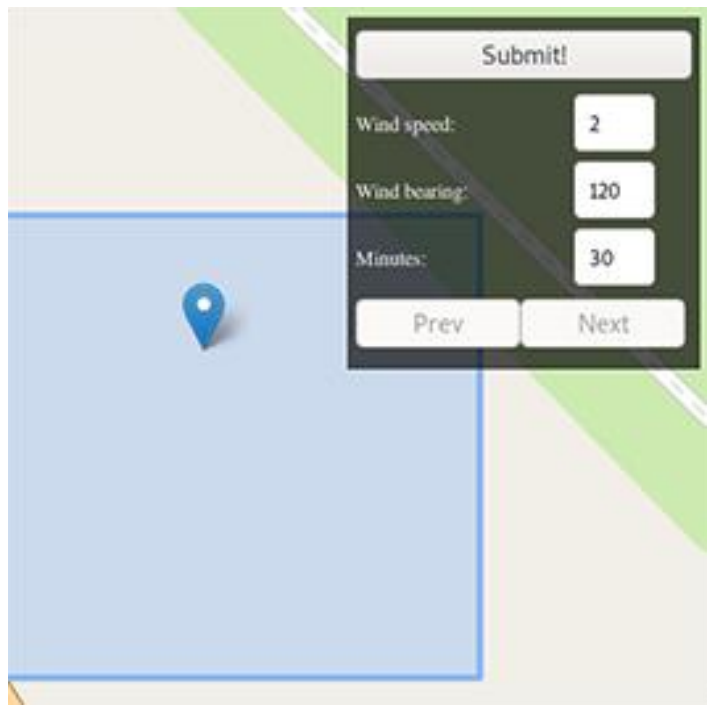

Figure 6: Simplified user interface, simulation extent (blue polygon), and fire origin (blue marker) 


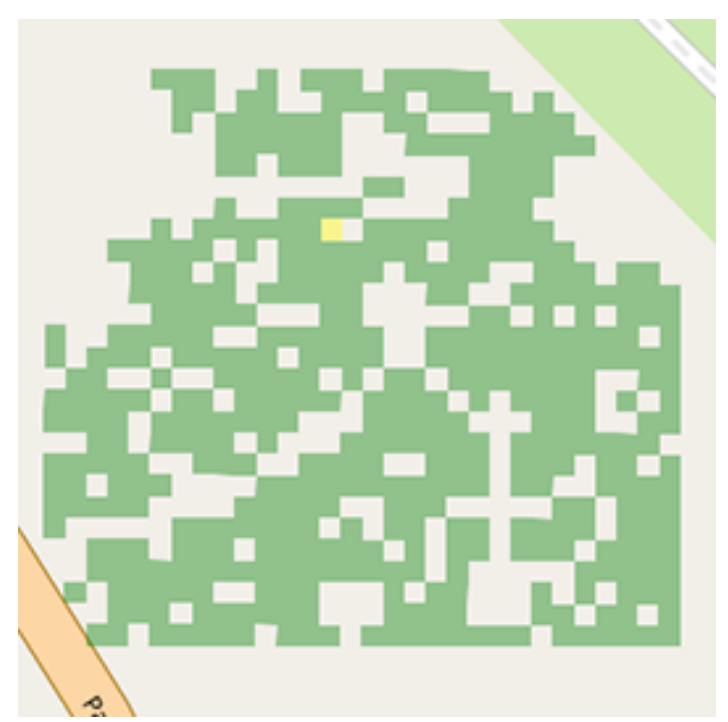

Figure 7: Simulation 1 minute in

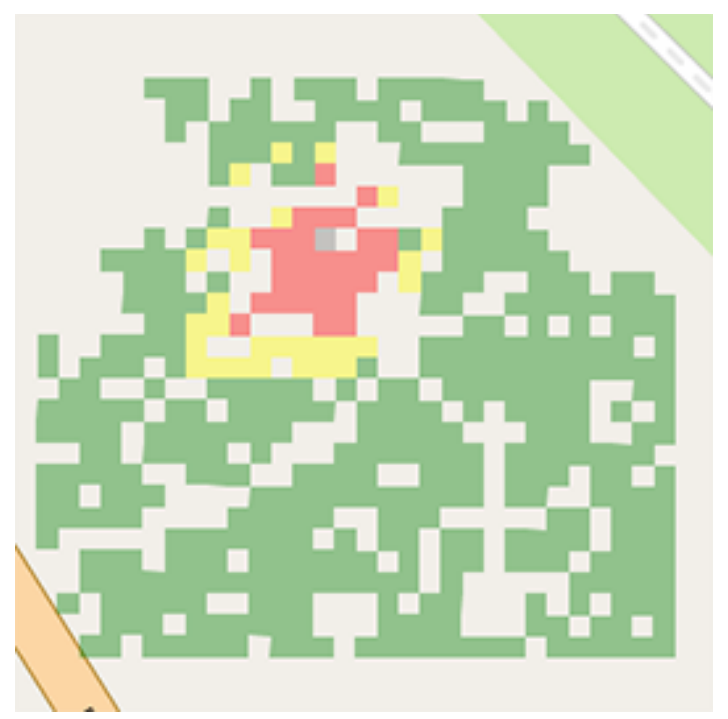

Figure 8: Simulation 15 minutes in (state [1] in green, state [2] in yellow, state [3] in red, state [4] in gray)

The simulation extent and fire origin are easily plottable on a web map via the UI; wind parameters and time limit are also configurable from the UI. Wind bearing is the direction of the wind in angle degrees (with $0^{\circ}$ as $\mathrm{E}, 90^{\circ}$ as $\mathrm{S}, 180^{\circ}$ as $\mathrm{W}$, and $270^{\circ}$ as N). The "Submit!" button sends the inputs to a server, where it is processed and the simulation is calculated. When the simulation is ready, the "Prev" and "Next" buttons move the simulation back and forth in time. Figure 7 and Figure 8 shows the simulation results 1 minute and 15 minutes in, respectively.

\section{MODEL EVALUATION}

The model evaluation is done by performing the simulation on past fire-stricken areas. Data such as the origin of fire, fire extent, and historical wind parameters of these fire-stricken areas were collected by the FireCheck Project. The result of the simulation is compared to the actual spread of fire. Two measures were used to evaluate the model: accuracy and the Cohen's kappa.

\subsection{Accuracy}

Accuracy is a test of how well the model identifies between true and false. It measures the ratio between correct classifications over all classification attempts. It is defined by the given formula:

$$
A=\frac{\mathrm{TP}+\mathrm{TN}}{\mathrm{TP}+\mathrm{TN}+\mathrm{FP}+\mathrm{FN}}
$$

where TP is true positive, TN is true negative, FP is false positive, and $\mathrm{FN}$ is false negative.

In the case of our fire spread model, we are to identify whether a cell is burnt or not burnt. We count a true positive if a cell (i, $\mathrm{j}$ ) is burnt in both the actual and the simulated. Further, we count a false positive if a cell $(i, j)$ is burnt in the simulation but is actually not burnt in the actual.

\subsection{Cohen's kappa}

The Cohen's kappa is another test used to measure the degree of agreement among "raters". This takes into account the possibility of both raters coming into agreement due to chance. Cohen's kappa is formulated as:

$$
\kappa=1-\frac{1-p_{o}}{1-p_{e}}
$$

where $p_{o}$ is the observed agreement (accuracy) and $p_{e}$ is the probability of chance agreement. The probability of chance agreement $p_{e}$ is calculated by first calculating the probability that both raters would agree by random. Suppose that the ratio of one rater $r_{1}$ says yes is 0.6 and the ratio of rater $r_{2}$ says yes is 0.3 , the probability that both would say yes at random is $p_{\text {yes }}=0.8 \times 0.5=0.4$ The calculation is performed similarly for the probability that both would say no $p_{n o}$. The overall probability of chance agreement is then the summation of both probabilities:

$$
p_{e}=p_{y e s}+p_{n o}
$$

In our case, our 2 raters are the actual fire and the simulated fire. Additionally, this test factors in the possibility of our model only being correct due to chance.

\subsection{Simulation Results}

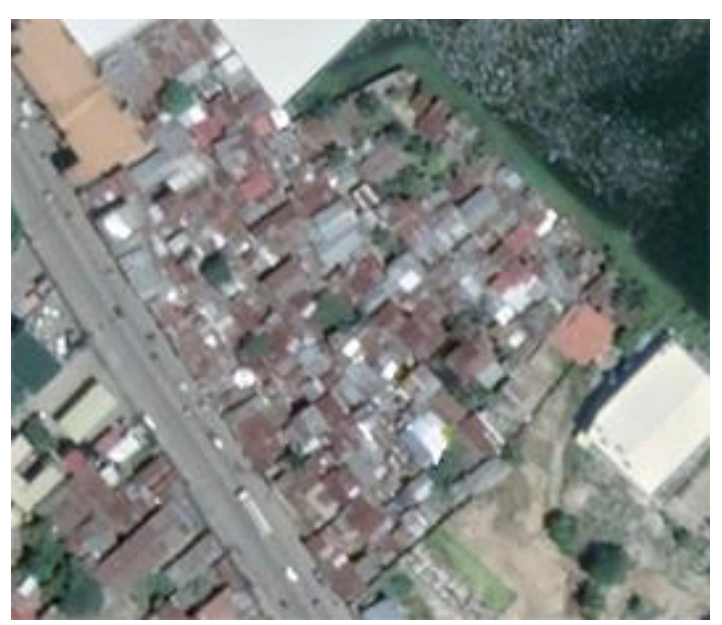

Figure 9: Tamiya, Barangay Basak, Lapu-lapu City (pre-fire) 
Sitio Santo Niño, commonly known as Tamiya, in barangay Basak, Lapu-lapu City (Figure 9) was chosen as the subject area of our evaluation. On March 20, 2018, fire struck the area destroying more than 300 houses. Since the buildings have already been burned down, the building material attributes of the area could not be collected. Instead, we carried over the distribution of material types from a similar area. This is done by collating the distribution of material types and heights from a visually similar area. The proportion of material type is then applied to the subject area.

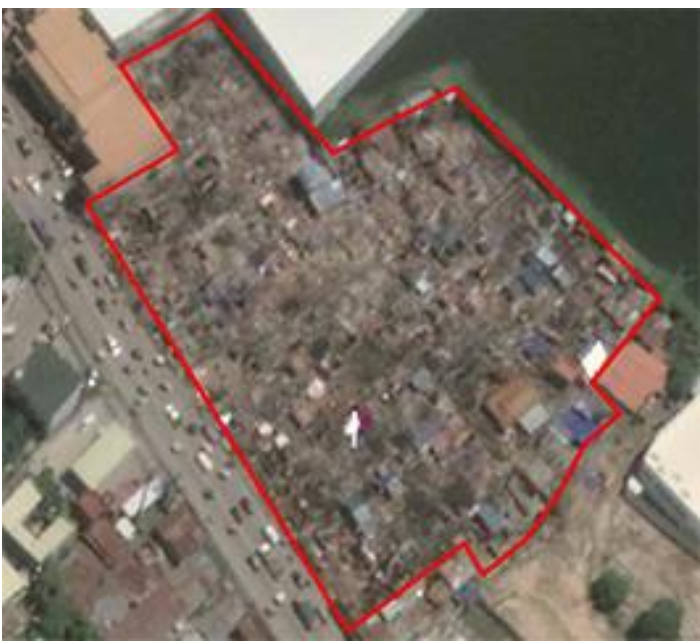

Figure 10: Tamiya, Barangay Basak, Lapu-lapu City (postfire, extent outlined in red)

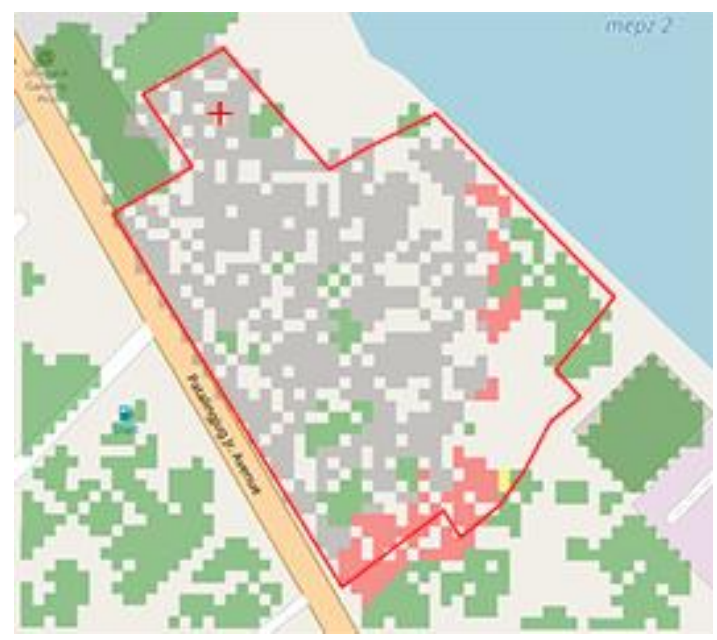

Figure 11: Completed fire spread simulation (actual extent outlined in red, fire origin marked by ' + ')

Based on the collected data from the FireCheck Project, the fire lasted around 77 minutes with the wind blowing at $2 \mathrm{~m} / \mathrm{s}$ towards the SW (bearing of $120^{\circ}$ ). With an $\alpha$ of 1, Figure 11 shows the simulated fire spread over the same area. The model achieved an $83.54 \%$ accuracy score with a Cohen's kappa of 0.67 . Visually, the simulated fire spread misses a few cells on the edge of the extent which contributes to the decrease of accuracy.

\section{CONCLUSIONS}

A fire spread model using matrices and cellular automata was developed, with an accuracy of $83.54 \%$. This proves that cellular automata can be used as an appropriate tool in modeling fire spread. Further work into properly assigning the building structure parameter of each cell can be done. The developed platform makes using the model accessible for use cases such as in evacuation planning. Further work can be done to improve the platform, such as displaying digitized buildings and improving the speed at which it processes the simulation.

\section{REFERENCES}

Almeida, R.M., Macau, E.E.N., 2011. Stochastic cellular automata model for wildland fire spread dynamics. Journal of Physics: Conference Series 285, 012038.

https://doi.org/10.1088/1742-6596/285/1/012038

Bhargava, S.C., Kumar, A., Mukherjee, A., 1993. A stochastic cellular automata model of innovation diffusion. Technological Forecasting and Social Change 44, 87-97. https://doi.org/10.1016/0040-1625(93)90008-U

Brieger, L., Bonomi, E., 1991. A stochastic cellular automation simulation of the non-linear diffusion equation. Physica D: Nonlinear Phenomena 47, 159-168. https://doi.org/10.1016/0167-2789(91)90287-J

Carmel, Y., Paz, S., Jahashan, F., Shoshany, M., 2009. Assessing fire risk using Monte Carlo simulations of fire spread. Forest Ecology and Management 257, 370-377. https://doi.org/10.1016/j.foreco.2008.09.039

Chopard, B., 2012. Cellular Automata Modeling of Physical Systems, in: Meyers, R.A. (Ed.), Computational Complexity. Springer New York, New York, NY, pp. 407-433. https://doi.org/10.1007/978-1-4614-1800-9_27

Galarpe, L., 2019. More than 1K fires in Central Visayas in 2018. Philippine News Agency.

Himoto, K., Shinohara, M., Sekizawa, A., Takanashi, K.-i., Saiki, H., 2018. A field experiment on fire spread within a group of model houses. Fire Safety Journal 96, 105-114. https://doi.org/10.1016/j.firesaf.2018.01.003

Himoto, K., Tanaka, T., 2008. Development and validation of a physics-based urban fire spread model. Fire Safety Journal 43, 477-494.

https://doi.org/10.1016/j.firesaf.2007.12.008

Karafyllidis, I., Thanailakis, A., 1997. A model for predicting forest fire spreading using cellular automata. Ecological Modelling 99, 87-97. https://doi.org/10.1016/S0304-3800(96)01942-4

Lee, S.W., Davidson, R.A., 2010. Physics-Based Simulation Model of Post-Earthquake Fire Spread. Journal of Earthquake Engineering 14, 670-687. https://doi.org/10.1080/13632460903336928

Ohgai, A., Gohnai, Y., Watanabe, K., 2007. Cellular automata modeling of fire spread in built-up areas-A tool to aid community-based planning for disaster mitigation. Computers, Environment and Urban Systems, Topology and Spatial Databases 31, 441-460.

https://doi.org/10.1016/j.compenvurbsys.2006.10.001

Sullivan, A.L., 2009. Wildland surface fire spread modelling, 1990-2007. 3: Simulation and mathematical analogue models. Int. J. Wildland Fire 18, 387-403.

https://doi.org/10.1071/WF06144 